\title{
Entropy Analysis for MHD Generalised Couette Flow in a Composite Duct
}

\author{
Paresh Vyas and Ashutosh Ranjan \\ Department of Mathematics, University of Rajasthan, Jaipur 302004, India \\ Correspondence should be addressed to Paresh Vyas; pvyasmaths@yahoo.com \\ Received 31 July 2014; Revised 8 January 2015; Accepted 17 February 2015 \\ Academic Editor: Stoyan Nedeltchev
}

Copyright (C) 2015 P. Vyas and A. Ranjan. This is an open access article distributed under the Creative Commons Attribution License, which permits unrestricted use, distribution, and reproduction in any medium, provided the original work is properly cited.

\begin{abstract}
This paper presents entropy analysis of electrically conducting Newtonian fluid flow inside a horizontal composite duct. The upper impermeable wall of the duct moves with a uniform velocity while the lower wall is porous strata of finite thickness with impermeable bottom. The upper wall and the impermeable bottom are at constant temperature but at different temperatures. The duct is divided into two regions: Region I of clear fluid and Region II of fluid saturated porous layer. Momentum and thermal regimes for clear and porous regions are matched at clear fluid-porous interface by employing suitable matching conditions. The governing equations are solved analytically. Analytical solutions obtained for velocity and temperature are utilized to compute entropy generation. The effects of pertinent parameter on temperature distribution, entropy generation, and Bejan number are portrayed graphically and discussed.
\end{abstract}

\section{Introduction}

All real processes are irreversible, and a physical quantity termed entropy defined in the second law of thermodynamics is a pertinent measure of irreversibility of the systems. Entropy analysis is vital for optimizing devices that confront "thermodynamic imperfection" due to heat transfer, mass transfer, imposed magnetic field, radiative heat transfer, fluid flow irreversibilities, and so forth. It is known that entropy generated in systems destroys available work and thus reduces its efficiency. Bejan has shown in his pioneer works [1-3] that in convective heat transfer processes one can identify parameters which are decisive in entropy generation. $\mathrm{He}$ emphasised that the combined heat transfer and thermodynamics model "portrays" irreversible nature of the device and thus the optimization of such a model gives us a feel for the otherwise abstract concept of entropy generation, specifically where and how much of it is being generated, how it flows, and how it impacts thermodynamic performance. Entropy analysis has proved to be a pertinent technique to help thermodynamic optimization emerge as a self-standing research area.
In the present scenario where energy optimization is a natural want there is much scope for devising optimal industrial thermal systems following second law analysis. Such analysis helps peep into minimization of entropy by simply identifying and selecting the parameters to eradicate the erosion of available energy for direct conversion to work. Once such pertinent information is available then it can be harnessed to design optimal thermal systems. Recently, entropy analysis is gaining currency in engineering fields like heat exchangers, cooling of nuclear reactors, energy storage systems, cooling of electronic devices, pumps, turbine, and pipe networks, and so forth. Last decade has witnessed a great surge of research activity in entropy generation aspects in fluid flow systems. These investigations were carried out theoretically in idealized flow configurations having technological implications.

Channel flow is one of the idealized configurations in fluid mechanics which serves as an important introductory model to large scale analogous systems. Some studies have been reported on the entropy analysis in channel flow. However, a rapid search of the literature reveals that there are three types of problems pertaining to entropy analysis for 
convective flow inside channels: firstly, channels where the fluid is clear; secondly, channels filled with fluid saturated porous material; thirdly, fluid flow in channels with permeable walls.

The inherent irreversibility of fluid flow and heat transfer for non-Newtonian power law fluids in a pipe and channel made of two parallel plates was investigated by Mahmud and Fraser [4]. Mahmud et al. [5] examined the entropy generation in a MHD mixed convection channel flow. Yilbas et al. [6] presented an analytical solution for entropy generation in a constant viscosity non-Newtonian third grade fluid flow in an annular pipe. They showed that entropy generation number attains high values in the region close to the inner wall of the annular pipe and increasing Brinkman number enhances entropy generation. Haddad et al. [7] examined entropy generation due to forced convection in parallel plates microchannel. Mahmud and Andrew Fraser [8] furnished a numerical treatment to entropy generation characteristics inside a porous channel considering viscous dissipation effect. Aziz [9] examined entropy generation in pressure gradient assisted Couette flow. Hooman and Ejlali [10] dealt with entropy production for thermally developing dissipative forced convection in a porous tube. Hooman et al. [11] studied entropy generation for forced convection in a porous channel with isoflux or isothermal walls. Makinde and Aziz [12] performed analytical and numerical analysis of the second law of thermodynamics for plane Poiseuille flow with asymmetric convective heat transfer taking variable fluid viscosity. Makinde [13] conducted entropy generation study in a falling variable viscosity liquid film along an inclined heated plate with convective cooling. Makinde and Bég [14] performed the second law analysis to the problem of inherent irreversibility in a reactive hydromagnetic channel flow. Makinde and Eegunjobi [15] investigated effects of convective heating on entropy generation rate in a channel with permeable walls. Vyas and Rai [16] investigated entropy regime for radiative MHD flow inside a channel with naturally permeable wall. Chauhan and Kumar [17-19] performed entropy analysis for third grade fluid.

Couette flow and associated thermal regime in a parallel plates channel involving porous substrate simulates flow and heat transfer behaviour of lubricants in porous journal bearings and porous rollers, geophysical channels, granular media filtration, and so forth. The thermal characteristics at clear fluid-porous interface are important to understand numerous processes like pollutant dispersion in aquifers, environment transport processes, separation processes in chemical industry, flow past porous scaffolds in bioreactors, drying process, and so forth. Many authors have investigated Couette flow for variety of configurations [20-25]. The laminarity of channel flow is a pertinent issue and therefore many numerical and laboratory studies have been devoted on the channel flow regime (clear fluid channel and porous medium channel) to be laminar. Newtonian fluid flow in the channel remains laminar when the pressure loss is directly proportional to the fluid velocity. Customarily, Reynolds number signifies the critical value beyond which the heat gradient is no longer proportional to the velocity. The critical value of Reynolds number as per experiments

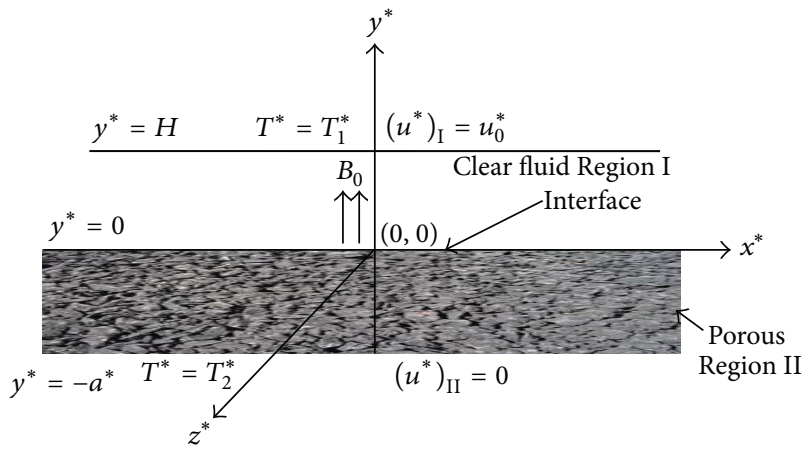

FIGURE 1: Flow configuration and coordinate systems.

ranges between 1 and 15 . Much insight into the issue can be seen in [26-29] and the references contained therein. In this backdrop, entropy generation analysis for MHD laminar Newtonian fluid flow and heat transfer in a composite duct is an interesting situation to look into.

\section{Formulation of the Problem}

A fully developed laminar flow of a viscous electrically conducting Newtonian incompressible fluid in a long horizontal composite duct of width $d=H+a$ is considered. The upper impermeable wall of the duct moves with a uniform velocity $u_{0}$ while the lower wall is stationary porous strata of finite thickness with impermeable bottom. The upper wall and the impermeable bottom are at constant but different temperatures $T_{1}^{*}$ and $T_{2}^{*}$, respectively $\left(T_{1}^{*}>T_{2}^{*}\right)$. The duct is divided into two regions: Region $\mathrm{I}\left(0 \leq y^{*} \leq H\right)$ consisting of clear fluid and Region II $\left(-a^{*} \leq y^{*} \leq 0\right)$ which is fluid saturated porous layer (Figure 1). A Cartesian coordinate system is used, where $O x^{*} y^{*} z^{*}$ constitutes a set of orthogonal axes with origin at the interface. The channel being parallel to $x^{*}-z^{*}$ plane is very long and is with large width in the $z^{*}$-direction; hence all the physical quantities except pressure depend on $y^{*}$ only.

A uniform magnetic field of strength $B_{0}$ is applied parallel to the $y^{*}$-axis. The induced magnetic field is neglected, which is valid for small magnetic Reynolds number. The fluid is assumed to be without phase change and in local thermal equilibrium with the porous medium. A constant pressure gradient $\partial p^{*} / \partial x^{*}$ at the mouth of the channel is also applied.

Considering the Brinkman model [30] for the porous medium, the governing equations for the setup under consideration are as follows:

$$
\begin{gathered}
\text { for Region I: }\left(0 \leq y^{*} \leq H\right) \\
\mu \frac{d^{2} u^{*}}{d y^{* 2}}-\sigma B_{0}^{2} u^{*}=\frac{\partial p^{*}}{\partial x^{*}}, \\
\kappa \frac{d^{2} T^{*}}{d y^{* 2}}+\mu\left(\frac{d u^{*}}{d y^{*}}\right)^{2}+\sigma B_{0}^{2} u^{* 2}=0 ;
\end{gathered}
$$


for Region II: $\left(-a^{*} \leq y^{*} \leq 0\right)$

$$
\begin{gathered}
\bar{\mu} \frac{d^{2} u^{*}}{d y^{* 2}}-\sigma B_{0}^{2} u^{*}-\frac{\mu}{k^{*}} u^{*}=\frac{\partial p^{*}}{\partial x^{*}}, \\
\bar{\kappa} \frac{d^{2} T^{*}}{d y^{* 2}}+\bar{\mu}\left(\frac{d u^{*}}{d y^{*}}\right)^{2}+\frac{\mu}{k^{*}} u^{* 2}+\sigma B_{0}^{2} u^{* 2}=0 .
\end{gathered}
$$

The quantities $u^{*}, p^{*}, \kappa, \mu, T^{*}, k^{*}$, and $\sigma$ denote the fluid velocity, pressure, thermal conductivity, coefficient of viscosity, temperature, permeability, and electrical conductivity, respectively. The quantities $\bar{\mu}$ and $\bar{\kappa}$ are effective viscosity and effective thermal conductivity, respectively, of the porous medium.

The boundary and interface conditions on velocity and temperature are as follows:

$$
\begin{gathered}
y^{*}=H: u^{*}=u_{0}^{*}, \quad T^{*}=T_{1}^{*} \\
y^{*}=0:\left(u^{*}\right)_{\mathrm{I}}=\left(u^{*}\right)_{\mathrm{II}}, \quad \mu \frac{d\left(u^{*}\right)_{\mathrm{I}}}{d y^{*}}=\bar{\mu} \frac{d\left(u^{*}\right)_{\mathrm{II}}}{d y^{*}}, \\
\left(T^{*}\right)_{\mathrm{I}}=\left(T^{*}\right)_{\mathrm{II}}, \quad \kappa \frac{d\left(T^{*}\right)_{\mathrm{I}}}{d y^{*}}=\bar{\kappa} \frac{d\left(T^{*}\right)_{\mathrm{II}}}{d y^{*}} \\
y^{*}=-a^{*}: u^{*}=0, \quad T^{*}=T_{2}^{*} .
\end{gathered}
$$

\section{Solution}

We introduce the following nondimensional quantities:

$$
\begin{gathered}
x=\frac{x^{*}}{H}, \quad y=\frac{y^{*}}{H}, \quad \quad \quad \quad=\frac{a^{*}}{H}, \quad u=\frac{u^{*} H}{v}, \\
u_{0}=\frac{u_{0}^{*} H}{v}, \quad p=\frac{p^{*} H^{2}}{\rho v^{2}}, \quad k=\frac{k^{*}}{H^{2}}, \\
\theta=\frac{T^{*}-T_{2}^{*}}{T_{1}^{*}-T_{2}^{*}}, \quad \frac{\partial p}{\partial x}=-C .
\end{gathered}
$$

In view of (6) the governing equations (1) through (4) take the following nondimensional forms:

for Region I $(0 \leq y \leq 1)$,

$$
\begin{gathered}
\frac{d^{2} u}{d y^{2}}-M^{2} u=-C, \\
\frac{d^{2} \theta}{d y^{2}}+\operatorname{Br}\left(\frac{d u}{d y}\right)^{2}+\operatorname{Br} M^{2} u^{2}=0
\end{gathered}
$$

for Region II $(-a \leq y \leq 0)$,

$$
\begin{gathered}
\frac{d^{2} u}{d y^{2}}-\phi_{1}\left(M^{2}+\frac{1}{k}\right) u=-C \phi_{1}, \\
\frac{d^{2} \theta}{d y^{2}}+\frac{\phi_{2}}{\phi_{1}} \operatorname{Br}\left(\frac{d u}{d y}\right)^{2}+\phi_{2} \operatorname{Br}\left(M^{2}+\frac{1}{k}\right) u^{2}=0
\end{gathered}
$$

The boundary conditions (5) in nondimensional form are reduced to the following:

$$
\begin{gathered}
y=1: u=u_{0}, \quad(\theta)_{\mathrm{I}}=1, \\
y=0:(u)_{\mathrm{I}}=(u)_{\mathrm{II}}, \quad \phi_{1} \frac{d(u)_{\mathrm{I}}}{d y}=\frac{d(u)_{\mathrm{II}}}{d y}, \\
(\theta)_{\mathrm{I}}=(\theta)_{\mathrm{II}}, \quad \phi_{2} \frac{d(\theta)_{\mathrm{I}}}{d y}=\frac{d(\theta)_{\mathrm{II}}}{d y}, \\
y=-a:(u)_{\mathrm{II}}=0, \quad(\theta)_{\mathrm{II}}=0 .
\end{gathered}
$$

$\phi_{1}=\mu / \bar{\mu}, \phi_{2}=\kappa / \bar{\kappa}$, and $M=\sqrt{H^{2} \sigma B_{0}^{2} / \mu}, \mathrm{Br}=\mu \nu^{2} /$ $\kappa H^{2}\left(T_{1}-T_{2}\right)$ are the Hartmann number and Brinkman number, respectively.

The equations for velocity and temperature fields for both regions given in (7) through (10) are linear ordinary differential equations and hence are amenable to closed form analytical solutions. In view of the boundary conditions (11) we get the solutions as follows:

$$
\text { for Region I, }
$$

$$
(u)_{\mathrm{I}}=A_{1} e^{M y}+A_{2} e^{-M y}+\frac{C}{M^{2}}
$$

$$
\begin{aligned}
(\theta)_{\mathrm{I}}= & -\operatorname{Br}\left(\frac{A_{1}^{2}}{2} e^{2 M y}+\frac{A_{2}^{2}}{2} e^{-2 M y}+\frac{C^{2} y^{2}}{2 M^{2}}\right. \\
& \left.+\frac{2 C}{M^{2}}\left(A_{1} e^{M y}+A_{2} e^{-M y}\right)\right) \\
& +B_{1} y+B_{2}
\end{aligned}
$$

for Region II,

$$
\begin{gathered}
(u)_{\mathrm{II}}=A_{3} e^{R y}+A_{4} e^{-R y}+\frac{C \phi_{1}}{R^{2}} \\
(\theta)_{\mathrm{II}}=-\frac{\operatorname{Br} \phi_{2}}{\phi_{1}}\left(\frac{A_{3}^{2}}{2} e^{R y}+\frac{A_{4}^{2}}{2} e^{-R y}+\frac{C^{2} \phi_{1}^{2} y^{2}}{2 R^{2}}\right. \\
\left.+\frac{2 C \phi_{1}}{R^{2}}\left(A_{3} e^{R y}+A_{4} e^{-R y}\right)\right) \\
+B_{3} y+B_{4},
\end{gathered}
$$

where $A_{1}, A_{2}, A_{3}$, and $A_{4}$ and $B_{1}, B_{2}, B_{3}$, and $B_{4}$ are constants of integration and $R^{2}=\phi_{1}\left(M^{2}+1 / k\right)$. 
On applying boundary conditions (11) to (12) through (15) we get simultaneous equations involving the constants of integration as follows:

$$
\begin{aligned}
& A_{1} e^{M}+A_{2} e^{-M}=u_{0}-\frac{C}{M^{2}} \\
& A_{1}+A_{2}-A_{3}-A_{4}=C\left(\frac{\phi_{1}}{R^{2}}-\frac{1}{M^{2}}\right) \text {, } \\
& \phi_{1} M A_{1}-\phi_{1} M A_{2}-R A_{3}+R A_{4}=0 \text {, } \\
& A_{3} e^{-a R}+A_{4} e^{a R}=-\frac{C \phi_{1}}{R^{2}} \\
& -B_{1}-B_{2}=-1-\operatorname{Br}\left(\frac{A_{1}^{2}}{2} e^{2 M}+\frac{A_{2}^{2}}{2} e^{-2 M}+\frac{C^{2}}{2 M^{2}}\right. \\
& \left.+\frac{2 C}{M^{2}}\left(A_{1} e^{M}+A_{2} e^{-M}\right)\right) \\
& B_{2}-B_{4}=\operatorname{Br}\left(\frac{A_{1}^{2}}{2}+\frac{A_{2}^{2}}{2}+\frac{2 C}{M^{2}}\left(A_{1}+A_{2}\right)\right) \\
& -\frac{\operatorname{Br} \phi_{2}}{\phi_{1}}\left(\frac{A_{3}^{2}}{2}+\frac{A_{4}^{2}}{2}+\frac{2 C \phi_{1}}{R^{2}}\left(A_{3}+A_{4}\right)\right) \text {, } \\
& \phi_{2} B_{1}-B_{3}=\phi_{2} \operatorname{Br}\left(A_{1}^{2} M-A_{2}^{2} M+\frac{2 C}{M}\left(A_{1}-A_{2}\right)\right) \\
& -\frac{\operatorname{Br} \phi_{2}}{\phi_{1}}\left(A_{3}^{2} R-A_{4}^{2} R+\frac{2 C \phi_{1}}{R}\left(A_{3}-A_{4}\right)\right), \\
& a B_{3}-B_{4}=\frac{-\mathrm{Br} \phi_{2}}{\phi_{1}}\left(\frac{A_{3}^{2}}{2} e^{-2 a R}+\frac{A_{4}^{2}}{2} e^{2 a R}+\frac{C^{2} \phi_{1}^{2} a^{2}}{2 R^{2}}\right. \\
& \left.+\frac{2 C \phi_{1}}{R^{2}}\left(A_{3} e^{-a R}+A_{4} e^{a R}\right)\right) .
\end{aligned}
$$

The systems of (16) to (19) for the unknowns $A_{1}, A_{2}$, $A_{3}$, and $A_{4}$ and (20) to (23) for the unknowns $B_{1}, B_{2}, B_{3}$, and $B_{4}$ have been solved by MATLAB using the module LINSOLVE and these values are then utilized to get the solution of governing equations. After determining velocity and temperature distributions we take on entropy generation.

\section{Second Law Analysis}

The local volumetric rate of entropy generation in presence of magnetic field following Woods [31] for the setup is given by the following:

for Region I,

$$
S_{G}=\frac{\kappa}{T_{2}^{* 2}}\left(\frac{d T^{*}}{d y^{*}}\right)^{2}+\frac{\mu}{T_{2}^{*}}\left(\frac{d u^{*}}{d y^{*}}\right)^{2}+\frac{\sigma B_{0}^{2} u^{* 2}}{T_{2}^{*}}
$$

for Region II,

$$
S_{G}=\frac{\bar{\kappa}}{T_{2}^{* 2}}\left(\frac{d T^{*}}{d y^{*}}\right)^{2}+\frac{\bar{\mu}}{T_{2}^{*}}\left(\frac{d u^{*}}{d y^{*}}\right)^{2}+\frac{\sigma B_{0}^{2} u^{* 2}}{T_{2}^{*}}+\frac{\bar{\mu} u^{* 2}}{T_{2}^{*} k^{*}} .
$$

It is clear from (24)-(25) that various sources contribute in entropy generation: the first term in these equations shows the contribution of heat transfer to the entropy generation, the second term is the local entropy generation due to fluid friction, and the third term signifies the effect of magnetic field in the generation of entropy. The last term in (25) accounts for local entropy generation due to friction offered by porous medium.

In order to define the dimensionless entropy generation rate we prescribe the characteristic entropy generation rate, the dimensionless temperature difference, and the nondimensional number for entropy generation, respectively, as follows:

$$
S_{G 0}=\frac{\kappa\left(T_{1}^{*}-T_{2}^{*}\right)}{H^{2} T_{2}^{* 2}}, \quad N_{s}=\frac{S_{G}}{S_{G 0}}, \quad \Omega=\frac{T_{2}^{*}}{T_{1}^{*}-T_{2}^{*}} .
$$

Thus the nondimensional number for entropy generation $N_{s}$ for both regions is given by the following:

for Region I,

$$
N_{s}=\left(\frac{d \theta}{d y}\right)^{2}+\operatorname{Br} \Omega\left[\left(\frac{d u}{d y}\right)^{2}+M^{2} u^{2}\right]=N_{1}+N_{2}
$$

for Region II,

$$
\begin{aligned}
N_{s} & =\frac{1}{\phi_{2}}\left(\frac{d \theta}{d y}\right)^{2}+\frac{\operatorname{Br} \Omega}{\phi_{1}}\left[\left(\frac{d u}{d y}\right)^{2}+M^{2} u^{2} \phi_{1}+\frac{u^{2}}{k}\right] \\
& =N_{3}+N_{4},
\end{aligned}
$$

where

$$
\begin{aligned}
& N_{1}=\left(\frac{d \theta}{d y}\right)^{2}, \\
& N_{2}=\operatorname{Br} \Omega\left[\left(\frac{d u}{d y}\right)^{2}+M^{2} u^{2}\right], \\
& N_{3}=\frac{1}{\phi_{2}}\left(\frac{d \theta}{d y}\right)^{2}, \\
& N_{4}=\frac{B r \Omega}{\phi_{1}}\left[\left(\frac{d u}{d y}\right)^{2}+M^{2} u^{2} \phi_{1}+\frac{u^{2}}{k}\right] .
\end{aligned}
$$

The Bejan number, an important irreversibility parameter, can be defined for both regions as follows:

for Region I,

$$
\mathrm{Be}=\frac{N_{1}}{N_{1}+N_{2}}
$$

for Region II,

$$
\mathrm{Be}=\frac{N_{3}}{N_{3}+N_{4}} \text {. }
$$




\section{Results and Discussions}

The profiles for the temperature distribution, entropy generation distribution, and Bejan number have been drawn and discussed. It is to be noted that the dotted vertical line $(y=0)$ indicates the porous-clear fluid interface. Figures 2-6 display the effects of pertinent parameters on temperature distribution. Before analysing the figures, we wish to emphasise that the fluid flow undertaken is dissipative. There are three sources of dissipation: firstly the viscous dissipation (internal fluid friction); secondly the Ohmic dissipation which means the heat produced by the opposing Lorentz force; and thirdly the friction due to the resistance offered by the walls of porous matrix. From the physical consideration it is known that dissipation serves as a heat source to the system causing considerable quantitative and qualitative changes to thermal regime. In Figure 2, the temperature distribution for various values of $\mathrm{Br}$ is plotted. When $\mathrm{Br}=0$, temperature distribution is linear. For $\mathrm{Br}>0$, a parabolic distribution is superimposed on it; that increases by increasing $\mathrm{Br}$ and the magnetic field; it also decreases by the increasing permeability of the porous bed. For a given value of the temperature difference $T_{1}^{*}-T_{2}^{*}>$ 0 , heat flows from the upper wall of the fluid as long as $\mathrm{Br}$ does not exceed a certain value after which the maximum temperature shifts from the upper wall region to somewhere in middle region of the channel, and heat flows from the fluid to the upper wall. It is evident that temperature attains peak somewhere in the middle of the channel. Figure 2 exhibits the effect of Brinkman number on the temperature distribution. The figure reveals that the temperature increases with an increase in Br. Larger values of the Brinkman number are indicative of rather more frictional heating in the system thereby causing rise in the temperature. In fact, frictional heating serves as energy source to modify the thermal regime. Figure 3 displays the variation in temperature $\theta$ for varying values of permeability parameter $k$. It is revealed that the temperature rises with the increasing values of the permeability parameter. Figures 4 and 5 display the variation in temperature $\theta$ for varying values of viscosity ratio $\phi_{1}$ and thermal conductivity ratio $\phi_{2}$, respectively. The figures reveal that there is a substantial increase in temperature for the increasing values of $\phi_{1}$ and $\phi_{2}$. The effect of Hartmann number $M$ on the temperature field has been shown in Figure 6. The figure reveals that the temperature decreases in the duct with an increase in the values of $M$.

The variations in entropy and Bejan number for different values of the parameters involved have been displayed in Figures 7-20. These figures reveal sharp jumps in entropy generation number and Bejan number at the clear fluidporous interface. From these figures it is also found that entropy is large in regions adjacent to wall and then attains its minima somewhere in the middle of the channel. This may be attributed to the fact that, in the middle of the channel, velocity and temperature attain their peaks indicating vanishing velocity and temperature gradients. We may conclude that the walls of the channel serve as sources to entropy generation. Bejan number vanishes in the middle of the channel and then increases in the vicinity of upper plate. The reason behind

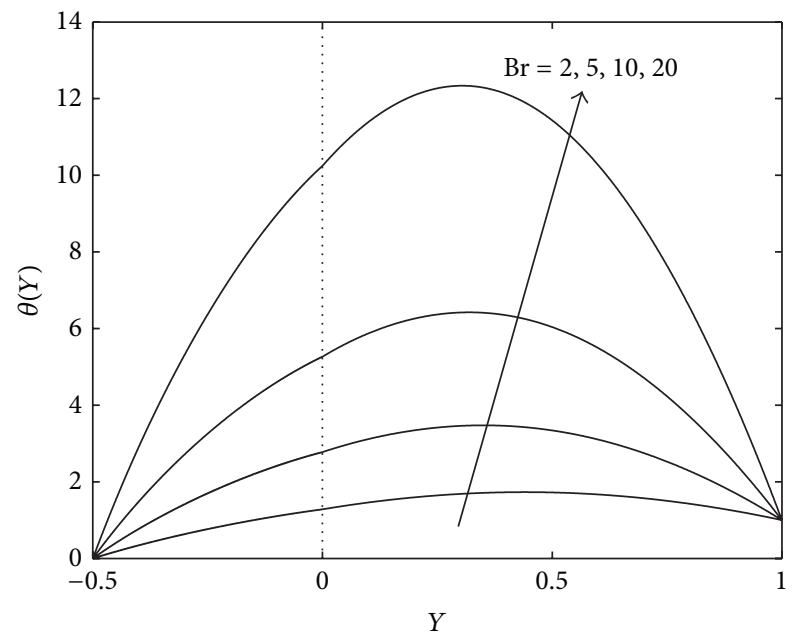

FIgURE 2: Temperature profiles for variation in $\mathrm{Br}$ when $M=2$, $k=0.5, u_{0}=0.5, a=0.5, C=5, \Omega=0.1, \phi_{1}=0.6$, and $\phi_{2}=0.8$.

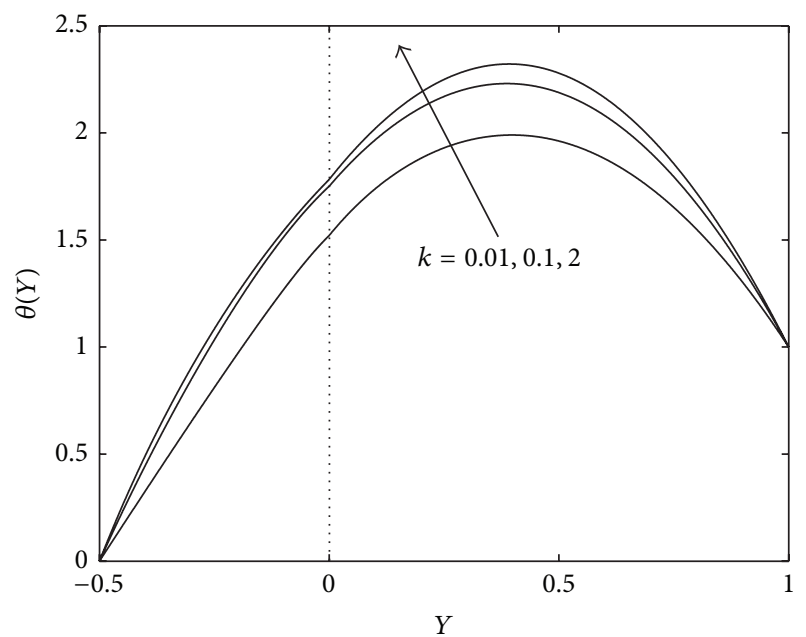

Figure 3: Temperature profiles for variation in $k$ when $M=2, \mathrm{Br}=$ $3, u_{0}=0.5, a=0.5, C=5, \Omega=0.1, \phi_{1}=0.6$, and $\phi_{2}=0.8$.

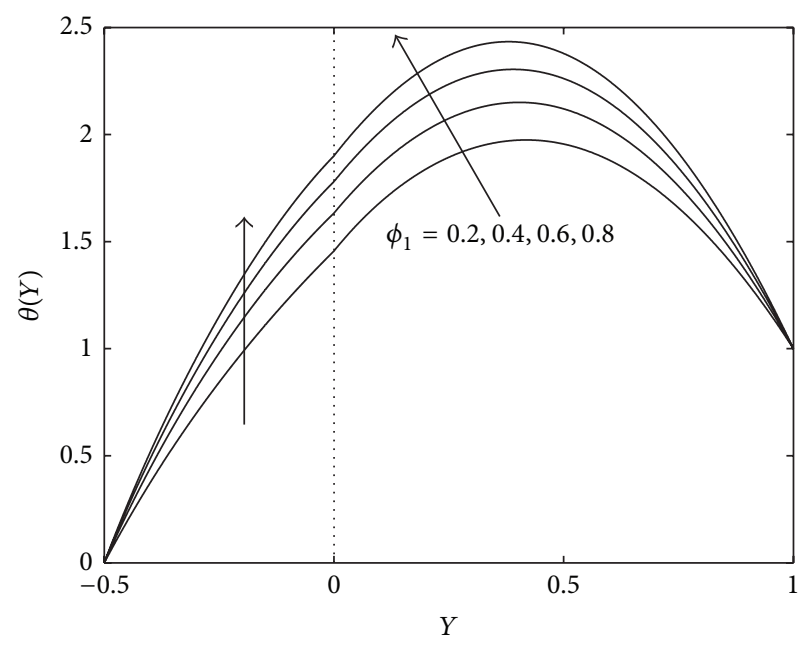

FIgURE 4: Temperature profiles for variation in $\phi_{1}$ when $M=2$, $k=0.5, u_{0}=0.5, a=0.5, C=5, \mathrm{Br}=3, \Omega=0.1$, and $\phi_{2}=0.8$. 


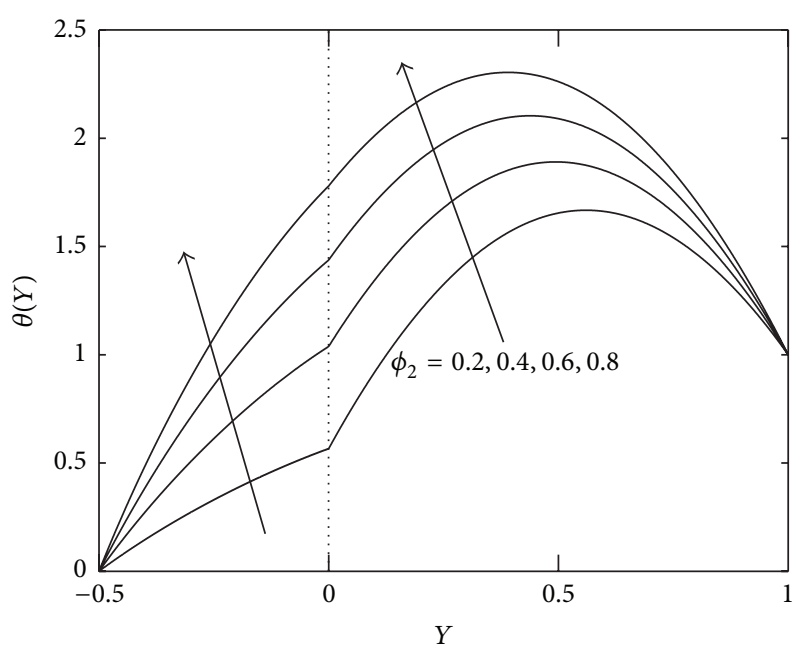

FIgURE 5: Temperature profiles for variation in $\phi_{2}$ when $M=2$, $k=0.5, u_{0}=0.5, a=0.5, C=5, \mathrm{Br}=3, \Omega=0.1$, and $\phi_{1}=0.6$.

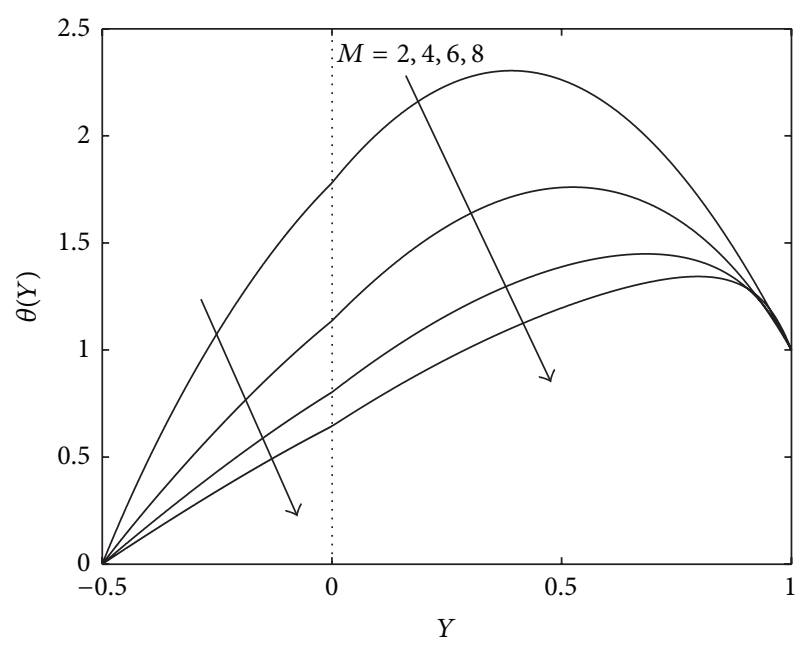

FIgURE 6: Temperature profiles for variation in $M$ when $k=0.5$, $\mathrm{Br}=3, u_{0}=0.5, a=0.5, C=5, \Omega=0.1, \phi_{1}=0.6$, and $\phi_{2}=0.8$.

this behaviour of Bejan number can be attributed to peaks in velocity and temperature in the middle of the channel, that is, vanishing velocity and temperature gradient.

Figures 7 and 8 display variations in entropy generation number and Bejan number, respectively, for varying values of Brinkman number Br. We find that, with the increasing values of $\mathrm{Br}$, entropy generation number increases. In fact larger $\mathrm{Br}$ values are indicative of more frictional heating in the system. Figure 9 reveals that, with the increasing values of permeability parameter $k$, entropy generation number $N_{s}$ increases in the regions close to the bottom while it decreases near the porous-clear fluid interface and further attains minima below the central line of the clear fluid region. The reason for this may be explained by the fact that larger values of permeability parameter $k$ indicate more fluid traversal inside porous medium. This results in lager friction due to internal fluid friction and the resistance offered by

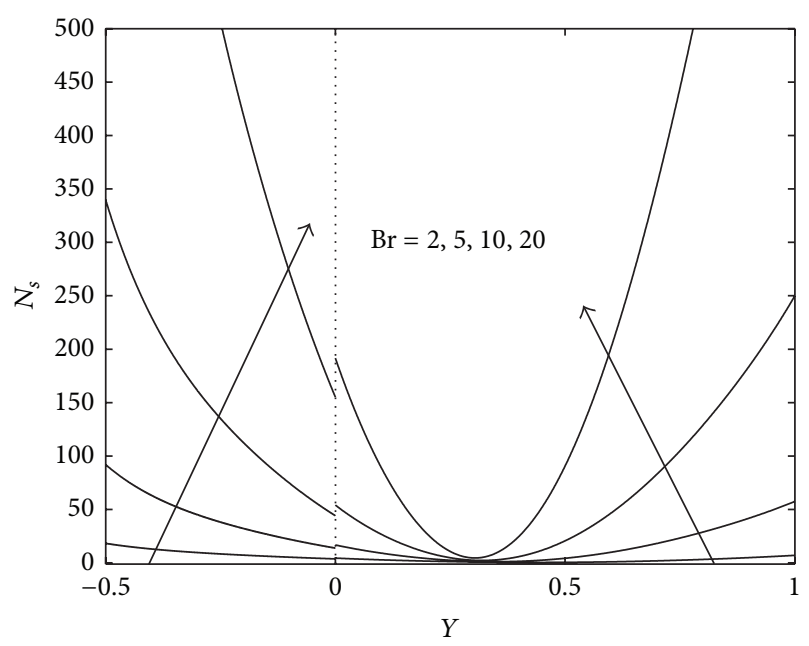

FIgURE 7: Entropy generation for variation in $\mathrm{Br}$ when $M=2, k=$ $0.5, u_{0}=0.5, a=0.5, C=5, \Omega=0.1, \phi_{1}=0.6$, and $\phi_{2}=0.8$.

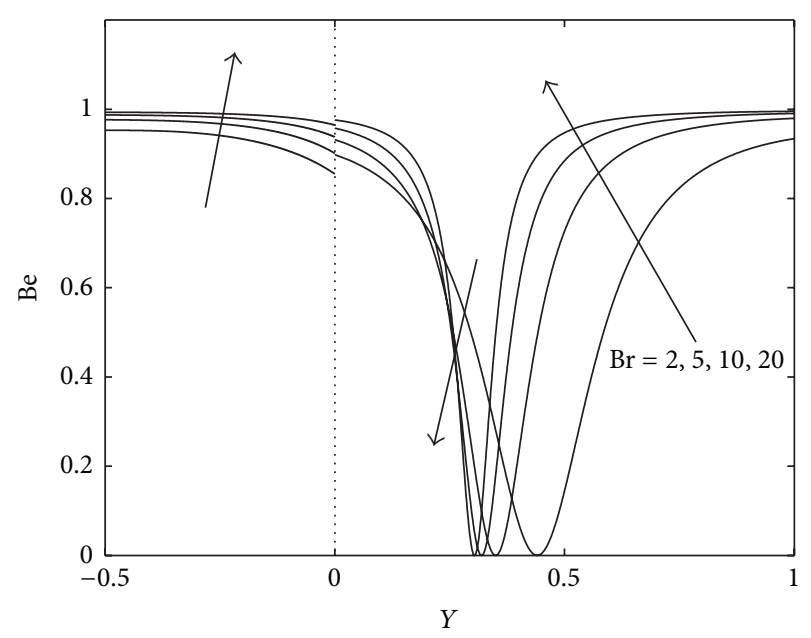

Figure 8: Bejan number for variation in $\operatorname{Br}$ when $M=2, k=0.5$, $u_{0}=0.5, a=0.5, C=5, \Omega=0.1, \phi_{1}=0.6$, and $\phi_{2}=0.8$.

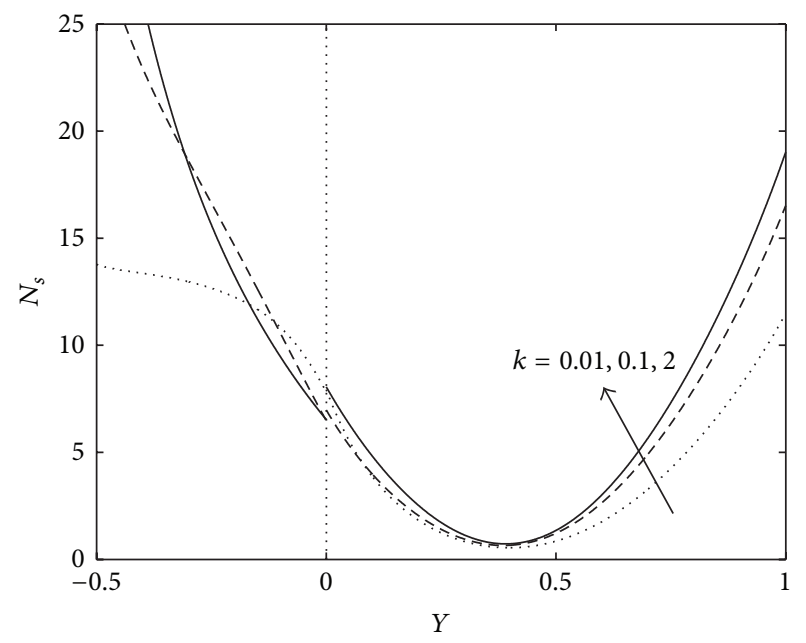

FIGURE 9: Entropy generation for variation in $k$ when $M=2, \mathrm{Br}=3$, $u_{0}=0.5, a=0.5, C=5, \Omega=0.1, \phi_{1}=0.6$, and $\phi_{2}=0.8$. 


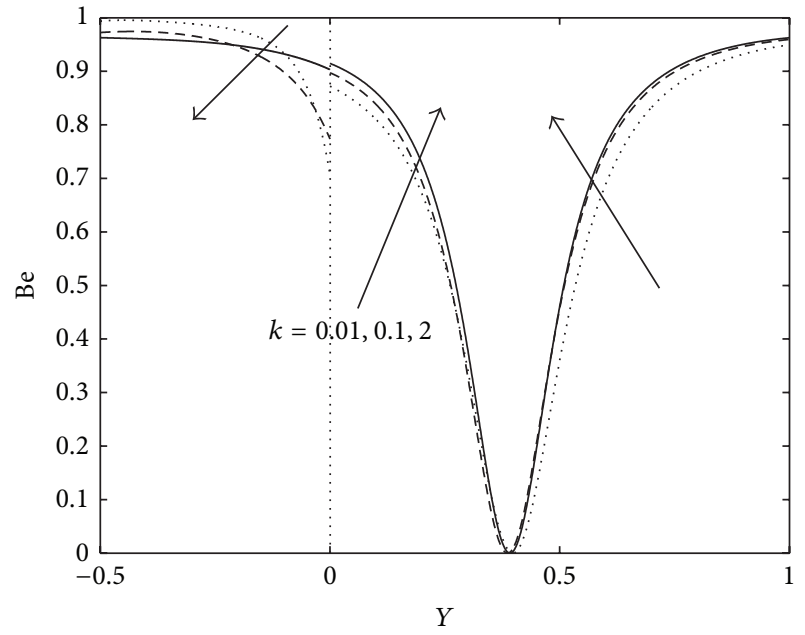

FIGURE 10: Bejan number for variation in $k$ when $M=2, \mathrm{Br}=3$, $u_{0}=0.5, a=0.5, C=5, \Omega=0.1, \phi_{1}=0.6$, and $\phi_{2}=0.8$.

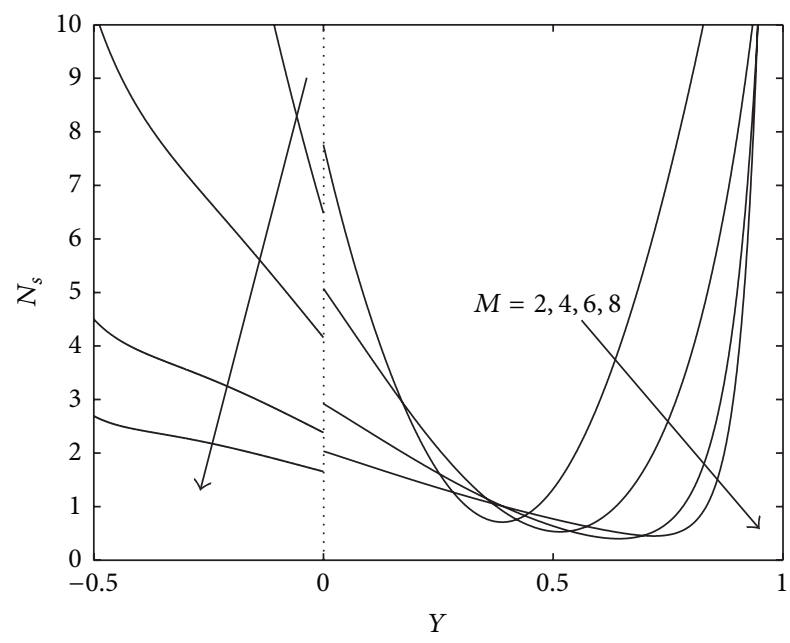

FIGURE 11: Entropy generation for variation in $M$ when $k=0.5$, $\mathrm{Br}=3, u_{0}=0.5, a=0.5, C=5, \Omega=0.1, \phi_{1}=0.6$, and $\phi_{2}=0.8$.

the porous medium walls. Figure 10 exhibits that Bejan number decreases near the lower wall and increases near the porous-clear fluid interface in porous region whereas it increases in clear fluid region with increasing values of $k$. Figure 11 depicts that, with the increasing values of $M$, entropy generation number decreases in the porous substrate, in regions adjacent to clear fluid-porous interface and the upper wall. Figures 12 and 13 display the effect of $M$ on Bejan number in clear fluid region and porous region, respectively. Figure 12 displays that, with the increasing values of $M$, the Bejan number Be increases considerably in the lower part of the clear region while the trend is reversed in the upper part of the channel. Be vanishes somewhere in the middle of the channel for respective values of $M$ in the porous region. However, for larger values of $M$, a reverse trend is observed in the middle of the porous region. Figure 13 reveals that variation in Be across the porous substrate for varying

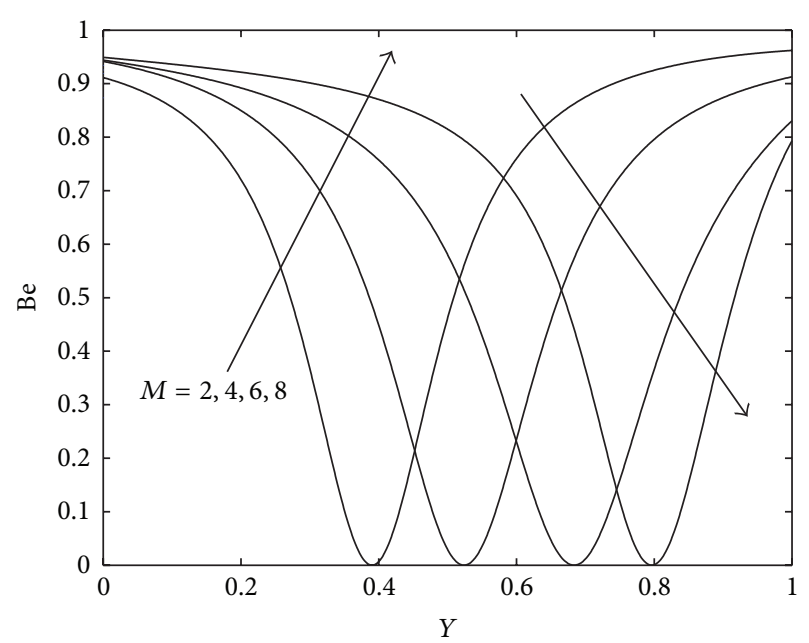

FIgURE 12: Bejan number for variation in $M$ when $k=0.5, \mathrm{Br}=3$, $u_{0}=0.5, a=0.5, C=5, \Omega=0.1, \phi_{1}=0.6$, and $\phi_{2}=0.8$ in Region I (R1).

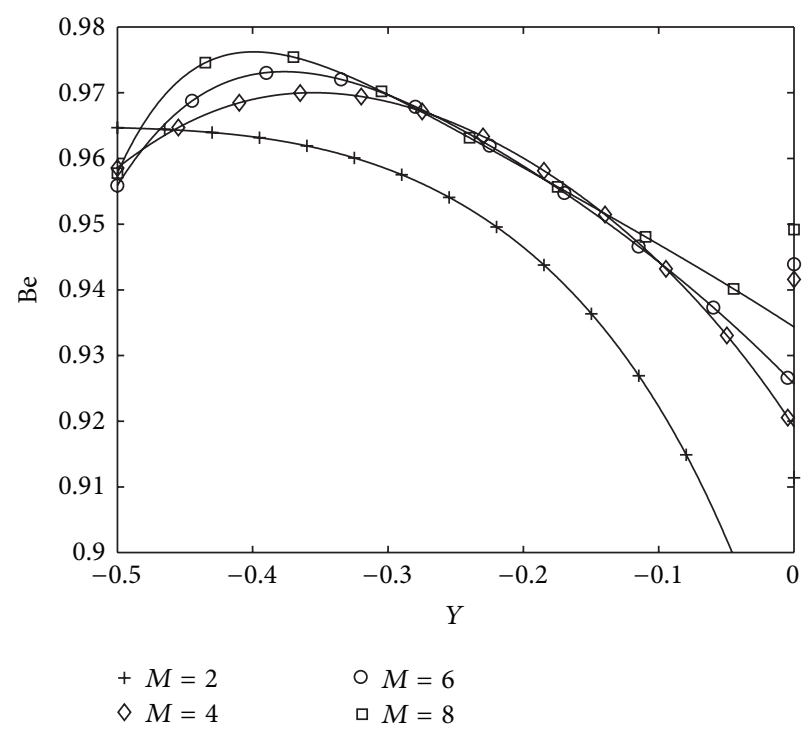

FIGURE 13: Bejan number for variation in $M$ when $k=0.5, \mathrm{Br}=3$, $u_{0}=0.5, a=0.5, C=5, \Omega=0.1, \phi_{1}=0.6$, and $\phi_{2}=0.8$ in Region II (R2).

$M$ is somewhat cumbersome. Figures 14 and 15 depict the effect of $\Omega$ on the entropy generation number and Bejan number, respectively. The figures reveal that $N_{s}$ increases and Be decreases throughout the channel considerably with the increasing values of $\Omega$. Figure 16 exhibits that an increment in viscosity ratio $\phi_{1}$ leads to an increment in $N_{s}$. Figures 17 and 18 reveal the effect of viscosity ratio $\phi_{1}$ on Bejan number in porous region and clear fluid region, respectively. Figure 17 displays that, with an increase in $\phi_{1}$, Bejan number decreases in the porous region except in the region adjacent to the porous-clear fluid interface where the trend is reversed. Figure 18 shows that, with the increasing values of $\phi_{1}$, the Bejan number decreases in the region $0.2 \leq y \leq 0.4$ and increases in the upper part of clear fluid region. It has been 


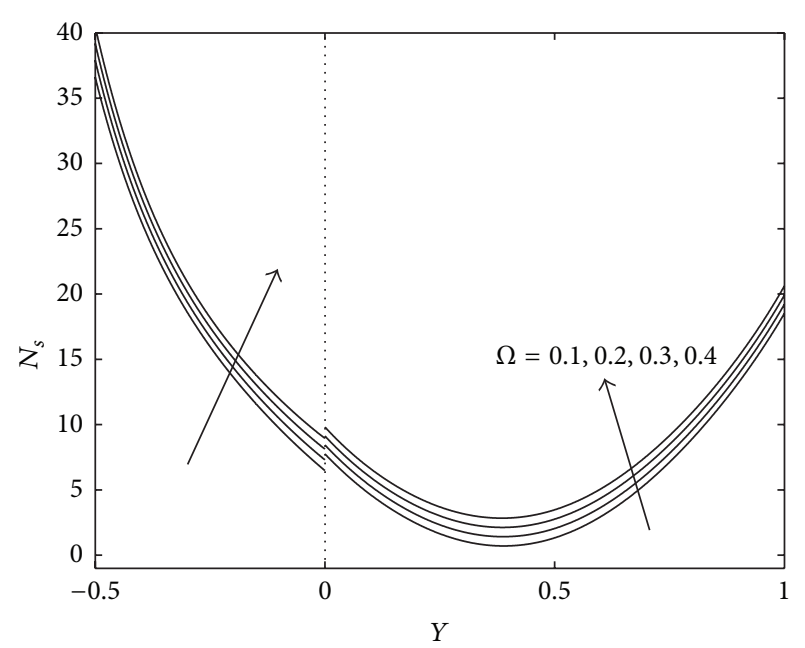

FIGURE 14: Entropy generation for variation in $\Omega$ when $M=2, \mathrm{Br}=$ $3, u_{0}=0.5, a=0.5, C=5, k=0.5, \phi_{1}=0.6$, and $\phi_{2}=0.8$.

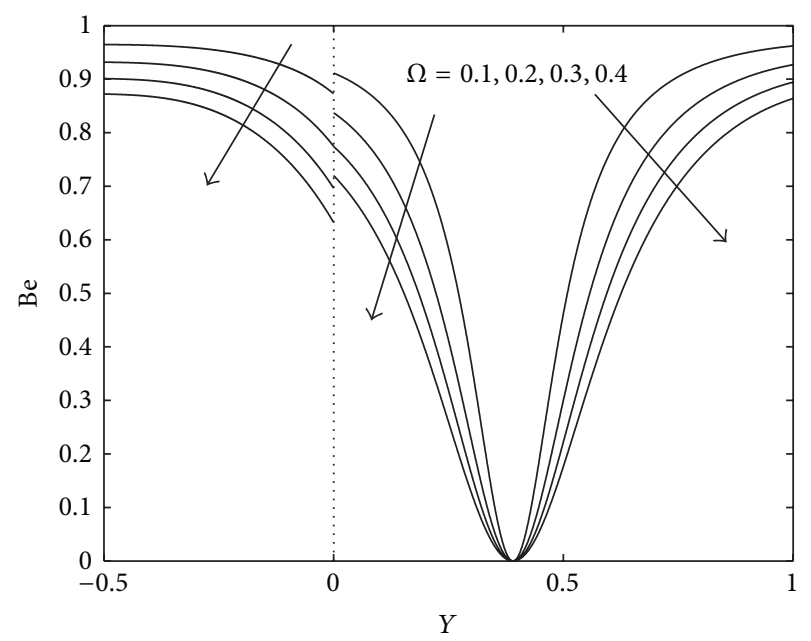

FIGURE 15: Bejan number for variation in $\Omega$ when $M=2, \mathrm{Br}=3$, $u_{0}=0.5, a=0.5, C=5, k=0.5, \phi_{1}=0.6$, and $\phi_{2}=0.8$.

observed that Be remains unchanged with the increasing values of $\phi_{1}$ at $y=0.2$. Figures 19 and 20 depict the effect of thermal conductivity ratio $\phi_{2}$ on the entropy generation number and Bejan number, respectively. The figure reveals that, with the increasing values of $\phi_{2}$, entropy increases in the porous region and in upper part of the clear fluid region; however the trend is reversed in the lower part of clear fluid region. The same phenomena are observed for the Bejan number for increasing values of $\phi_{2}$.

\section{Conclusion}

Generalised MHD Couette flow in a parallel composite channel is considered. The channel is bounded by an isothermal impermeable moving wall and a stationary porous substrate with impermeable bottom maintained at constant temperature different from that of the upper wall. The governing

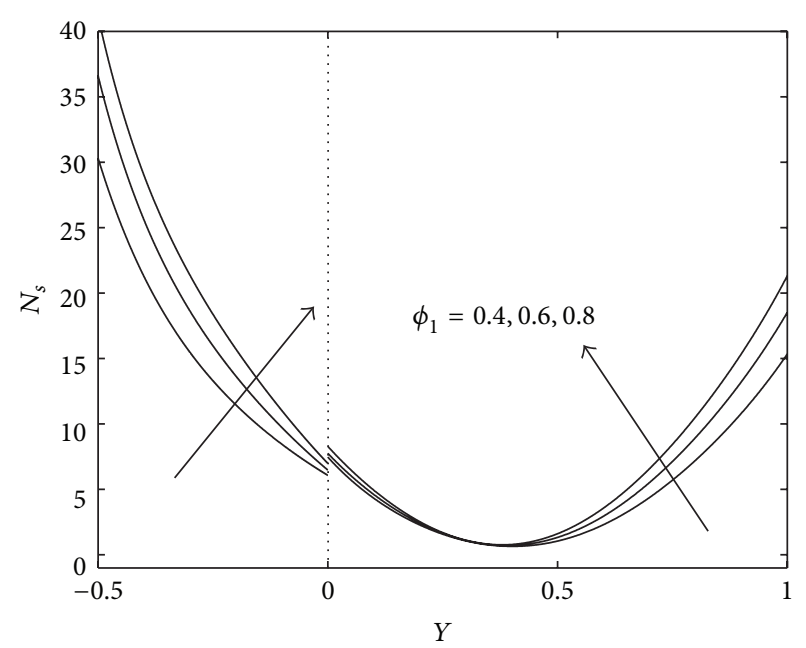

FIGURE 16: Entropy generation for variation in $\phi_{1}$ when $M=2, k=$ $0.5, u_{0}=0.5, a=0.5, C=5, \mathrm{Br}=3, \Omega=0.1$, and $\phi_{2}=0.8$.

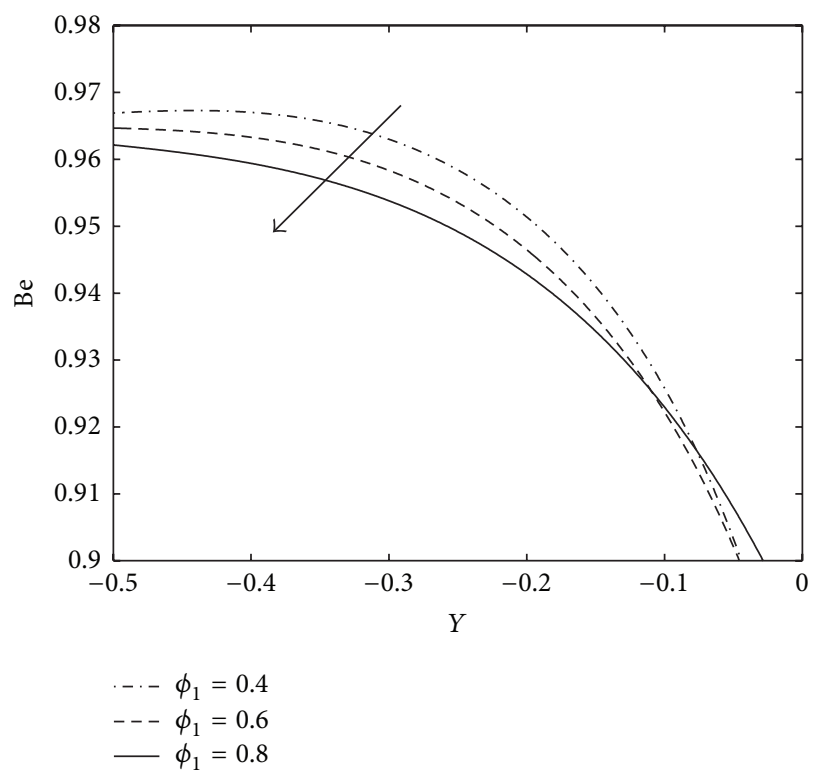

FIgURE 17: Bejan number for variation in $\phi_{1}$ when $M=2, k=0.5$, $u_{0}=0.5, a=0.5, C=5, \mathrm{Br}=3, \Omega=0.1$, and $\phi_{2}=0.8$ in Region II (R2).

equations for momentum and energy for the clear region and for the porous region are solved in view of the boundary conditions and matching conditions at the clear fluid- porous interface. Velocity and temperature distributions for both regions are derived analytically which are used to compute entropy generation. The effects of pertinent parameters on the quantities of interest are depicted graphically and discussed. The findings are as follows.

(1) Temperature in the channel increases with the increase in Brinkman number, permeability parameter, viscosity ratio $\phi_{1}$, and thermal conductivity ratio $\phi_{2}$ whereas it decays with the increasing values of Hartmann number $M$. 


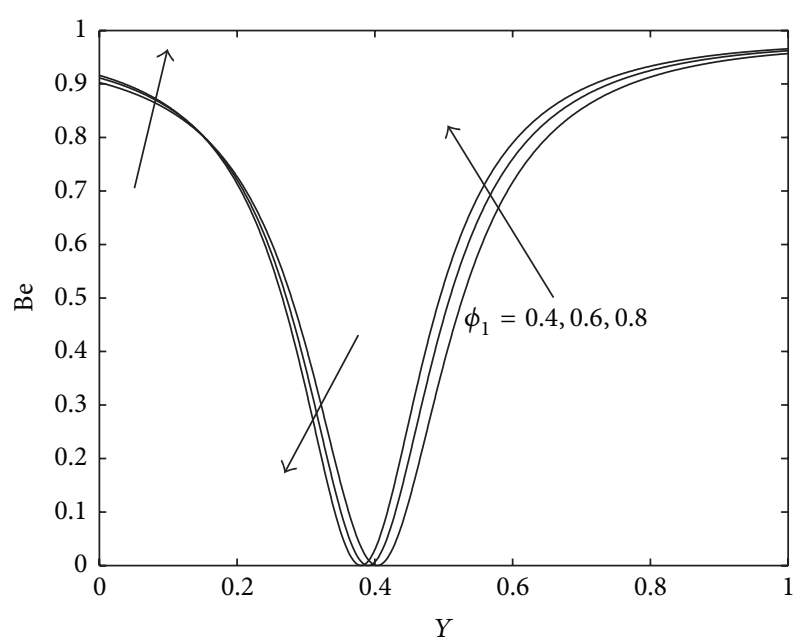

Figure 18: Bejan number for variation in $\phi_{1}$ when $M=2, k=0.5$, $u_{0}=0.5, a=0.5, C=5, \mathrm{Br}=3, \Omega=0.1$, and $\phi_{2}=0.8$ in Region I (R1).

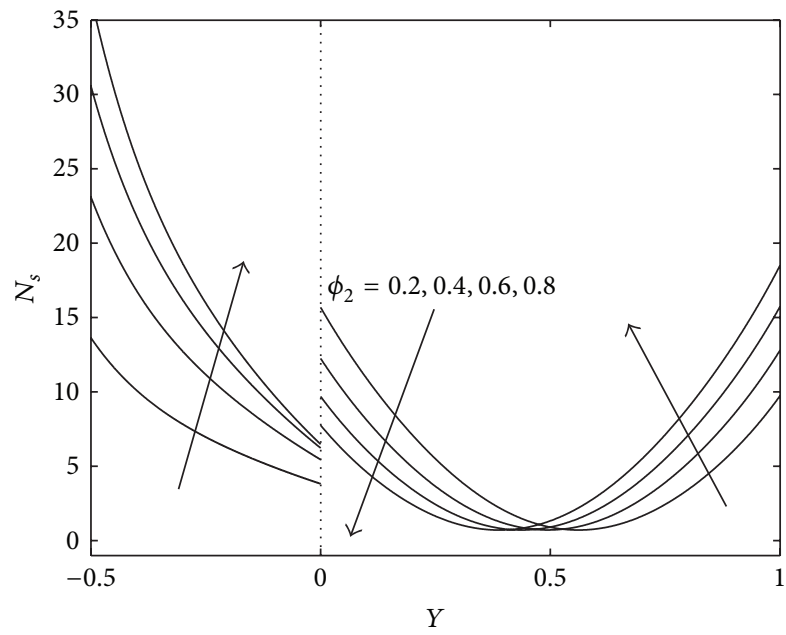

FIGURE 19: Entropy generation for variation in $\phi_{2}$ when $M=2, k=$ $0.5, u_{0}=0.5, a=0.5, C=5, \mathrm{Br}=3, \Omega=0.1$, and $\phi_{1}=0.6$.

(2) Entropy generation number rises in the clear and porous regions adjacent to walls with the increasing values of $\mathrm{Br}, k, \Omega, \phi_{1}$, and $\phi_{2}$ while the reverse trend is observed for the increasing values of $M$.

(3) The Bejan number rises with increasing values of $\mathrm{Br}$ in porous region, in the vicinity of clear fluid interface, and in the upper part of the channel.

(4) With the increasing values of permeability parameter $k$, Bejan number increases in the clear region. However the trend is reversed at the interface.

(5) Variation in Bejan number for varying values of $M$ is such that in clear region Be decreases in the upper part of the channel with the increasing values of $M$, while the trend is reversed in the lower part of clear region.

(6) Bejan number decays with the increasing values of $\Omega$.

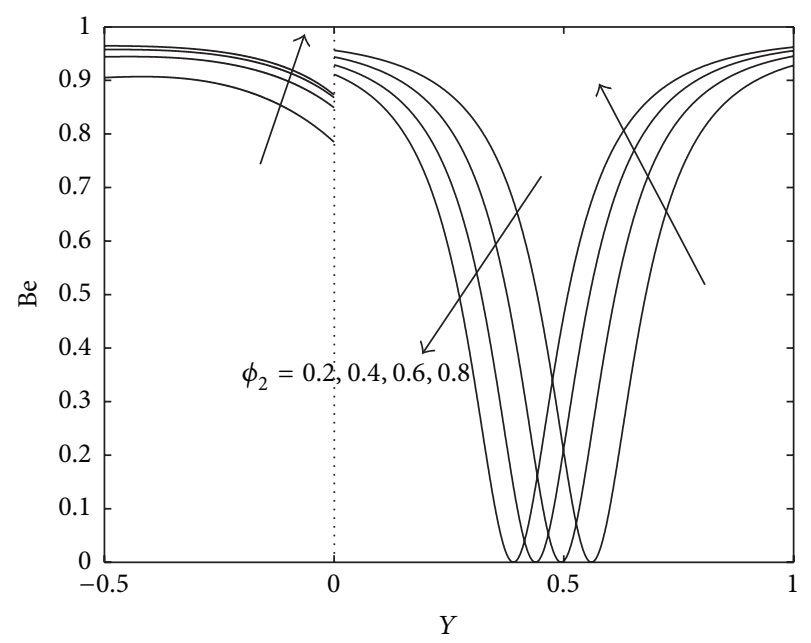

Figure 20: Bejan number for variation in $\phi_{2}$ when $M=2, k=0.5$, $u_{0}=0.5, a=0.5, C=5, \mathrm{Br}=3, \Omega=0.1$, and $\phi_{1}=0.6$.

(7) With the increasing values of $\phi_{1}$ and $\phi_{2}$, Bejan number increases in the regions close to the walls.

\section{Nomenclature}

$a: \quad$ Nondimensional thickness of porous medium

$a^{*}: \quad$ Thickness of porous medium

Be: Bejan number

$B_{0}$ : $\quad$ Magnetic field

Br: $\quad$ Brinkman number

$H: \quad$ Width of clear region

$k^{*}: \quad$ Permeability of the porous medium

$k: \quad$ Nondimensional permeability

$M$ : Hartmann number

$N_{s}: \quad$ Entropy generation number

$p^{*}: \quad$ Pressure

$S_{G}: \quad$ Entropy generation rate

$S_{G_{0}}: \quad$ Characteristic entropy generation rate

$T^{*}: \quad$ Temperature

$T_{1}^{*}, T_{2}^{*}: \quad$ Temperature of upper wall and impermeable bottom, respectively

$u^{*}: \quad$ Fluid velocity along $x^{*}$-direction

$U: \quad$ Nondimensional velocity

$O x^{*} y^{*} z^{*}$ : Coordinate system.

\section{Greek Letters}

$\theta: \quad$ Nondimensional temperature

$\kappa: \quad$ Thermal conductivity

$\bar{\kappa}$ : $\quad$ Effective thermal conductivity

$\mu$ : $\quad$ Fluid viscosity

$\bar{\mu}: \quad$ Effective viscosity

$\sigma: \quad$ Electrical conductivity

$\phi_{1}, \phi_{2}$ : Ratios of viscosity and thermal conductivity, respectively

$\Omega: \quad$ Dimensionless temperature difference. 


\section{Conflict of Interests}

The authors declare that there is no conflict of interests regarding the publication of this paper.

\section{References}

[1] A. Bejan, "A study of entropy generation in fundamental convective heat transfer," Journal of Heat Transfer, vol. 101, no. 4, pp. 718-725, 1979.

[2] A. Bejan, "Second-law analysis in heat transfer and thermal design," Advances in Heat Transfer, vol. 15, pp. 1-58, 1982.

[3] A. Bejan, Entropy Generation Minimization, CRC Press, Boca Raton, Fla, USA, 1996.

[4] S. Mahmud and R. A. Fraser, "Thermodynamic analysis of flow and heat transfer inside channel with two parallel plates," Exergy, vol. 2, no. 3, pp. 140-146, 2002.

[5] S. Mahmud, S. H. Tasnim, and M. A. H. Mamun, "Thermodynamic analysis of mixed convection in a channel with transverse hydromagnetic effect," International Journal of Thermal Sciences, vol. 42, no. 8, pp. 731-740, 2003.

[6] B. S. Yilbas, M. Yürüsoy, and M. Pakdemirli, "Entropy analysis for non-Newtonian fluid flow in annular pipe: constant viscosity case," Entropy, vol. 6, no. 3, pp. 304-315, 2004.

[7] O. Haddad, M. Abuzaid, and M. Al-Nimr, "Entropy generation due to laminar incompressible forced convection flow through parallel-plates microchannel," Entropy, vol. 6, no. 5, pp. 413-426, 2004.

[8] S. Mahmud and R. Andrew Fraser, "Flow, thermal, and entropy generation characteristics inside a porous channel with viscous dissipation," International Journal of Thermal Sciences, vol. 44, no. 1, pp. 21-32, 2005.

[9] A. Aziz, "Entropy generation in pressure gradient assisted Couette flow with different thermal boundary conditions," Entropy, vol. 8, no. 2, pp. 50-62, 2006.

[10] K. Hooman and A. Ejlali, "Entropy generation for forced convection in a porous saturated circular tube with uniform wall temperature," International Communications in Heat and Mass Transfer, vol. 34, no. 4, pp. 408-419, 2007.

[11] K. Hooman, F. Hooman, and S. R. Mohebpour, "Entropy generation for forced convection in a porous channel with isoflux or isothermal walls," International Journal of Exergy, vol. 5, no. 1, pp. 78-96, 2008.

[12] O. D. Makinde and A. Aziz, "MHD mixed convection from a vertical plate embedded in a porous medium with a convective boundary condition," International Journal of Thermal Sciences, vol. 49, no. 9, pp. 1813-1820, 2010.

[13] O. D. Makinde, "On MHD heat and mass transfer over a moving vertical plate with a convective surface boundary condition," Canadian Journal of Chemical Engineering, vol. 88, no. 6, pp. 983-990, 2010.

[14] O. D. Makinde and O. A. Bég, "On inherent irreversibility in a reactive hydromagnetic channel flow," Journal of Thermal Science, vol. 19, no. 1, pp. 72-79, 2010.

[15] O. D. Makinde and A. S. Eegunjobi, "Effects of convective heating on entropy generation rate in a channel with permeable walls," Entropy, vol. 15, no. 1, pp. 220-233, 2013.

[16] P. Vyas and A. Rai, "Entropy regime for radiative dissipative MHD couette flow inside channel with naturally permeable base," International Journal of Energy \& Technology, vol. 5, no. 19, pp. 1-9, 2013.
[17] D. S. Chauhan and V. Kumar, "Effects of slip conditions on forced convection and entropy generation in a circular channel occupied by a highly porous medium: darcy extended Brinkman-Forchheimer model," Turkish Journal of Engineering and Environmental Sciences, vol. 33, no. 2, pp. 91-104, 2009.

[18] D. S. Chauhan and A. Olkha, "Entropy generation and heat transfer effects on non-Newtonian fluid flow in annular pipe with naturally permeable boundaries," International Journal of Energy Technology, vol. 3, no. 30, pp. 1-9, 2011.

[19] D. S. Chauhan and V. Kumar, "Entropy analysis for third-grade fluid flow with temperature-dependent viscosity in annulus partially filled with porous medium," Theoretical and Applied Mechanics, vol. 40, no. 3, pp. 441-464, 2013.

[20] D. S. Chauhan and P. Vyas, "Heat transfer in hydromagnetic Couette flow of compressible Newtonian fluid," Journal of Engineering Mechanics, vol. 121, no. 1, pp. 57-61, 1995.

[21] A. V. Kuznetsov, "Analytical investigation of Couette flow in a composite channel partially filled with a porous medium and partially with a clear fluid," International Journal of Heat and Mass Transfer, vol. 41, no. 16, pp. 2556-2560, 1998.

[22] A. V. Kuznetsov, "Analytical investigation of heat transfer in Couette flow through a porous medium utilizing the BrinkmanFforchheimer-extended Darcy model," Acta Mechanica, vol. 129, no. 1-2, pp. 13-24, 1998.

[23] A. V. Kuznetsov, "Fluid flow and heat transfer analysis of couette flow in a composite duct," Acta Mechanica, vol. 140, no. 3, pp. 163-170, 2000.

[24] P. Vyas and N. Srivastava, "Radiation effects on dissipative magnetohydrodynamic couette flow in a composite channel," Zeitschrift fur Naturforschung-Section A, vol. 68, no. 8-9, pp. 554-566, 2013.

[25] P. Vyas and N. Srivastava, "Radiative MHD compressible Couette flow in a parallel channel with a naturally permeable wall," Thermal Science, vol. 18, supplement 2, pp. S573-S585, 2014.

[26] K. P. Stark, "A numerical study of the nonlinear laminar regime of flow in an idealized porous medium," in IAHR, Fundamentals of Transport Phenomena in Porous Media, pp. 86-102, Elsevier, Amsterdam, The Netherlands, 1972.

[27] J. Devries, "Prediction of non-Darcy flow in porous media," Journal of the Irrigation and Drainage Division, ASCE, vol. 105, no. 14610, pp. 147-162, 1979.

[28] A. Dybbs and R. V. Edwards, "A new look at porous media fluid mechanics-darcy to turbulent," in Fundamentals of Transport Phenomena in Porous Media, J. Bear and M. Y. Corapcioglu, Eds., pp. 199-256, Martinus Nijhoff Publishers, Dordrecht, The Netherlands, 1984.

[29] O. Coulaud, P. Morel, and J. Caltagirone, "Effets nonlinéaires les écoulments en milieu poreux," Comptes Rendus de l'Académie des Sciences, Paris-Series II, vol. 6, pp. 263-266, 1986.

[30] H. C. Brinkman, "A calculation of the viscous force exerted by a flowing fluid on a dense swarm of particles," Applied Scientific Research, vol. 1, no. 1, pp. 27-34, 1949.

[31] L. C. Woods, Thermodynamics of Fluid Systems, Oxford University Press, Oxford, UK, 1975. 


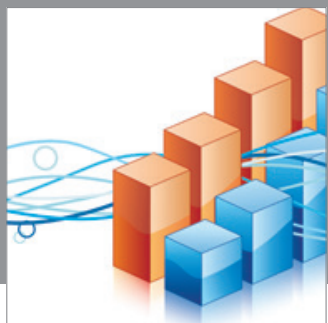

Advances in

Operations Research

mansans

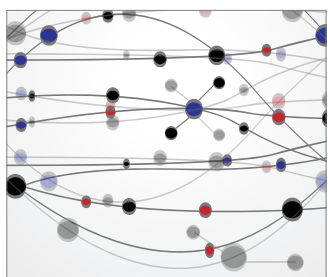

The Scientific World Journal
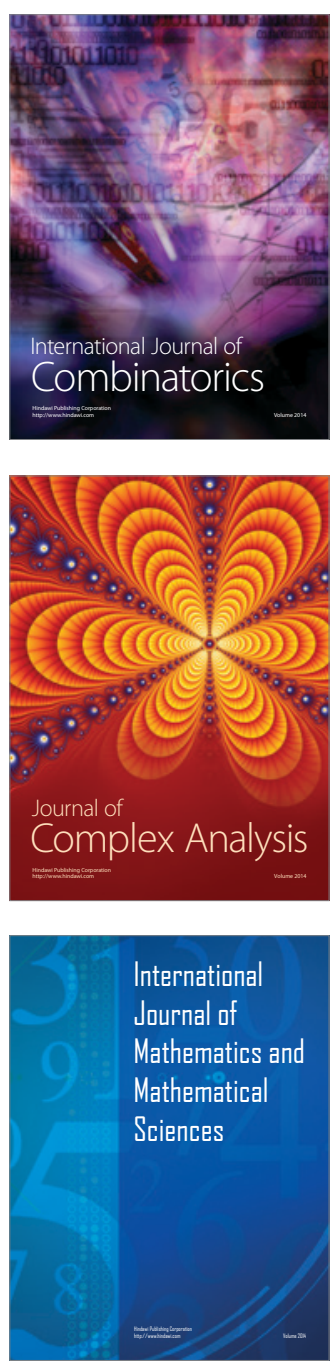
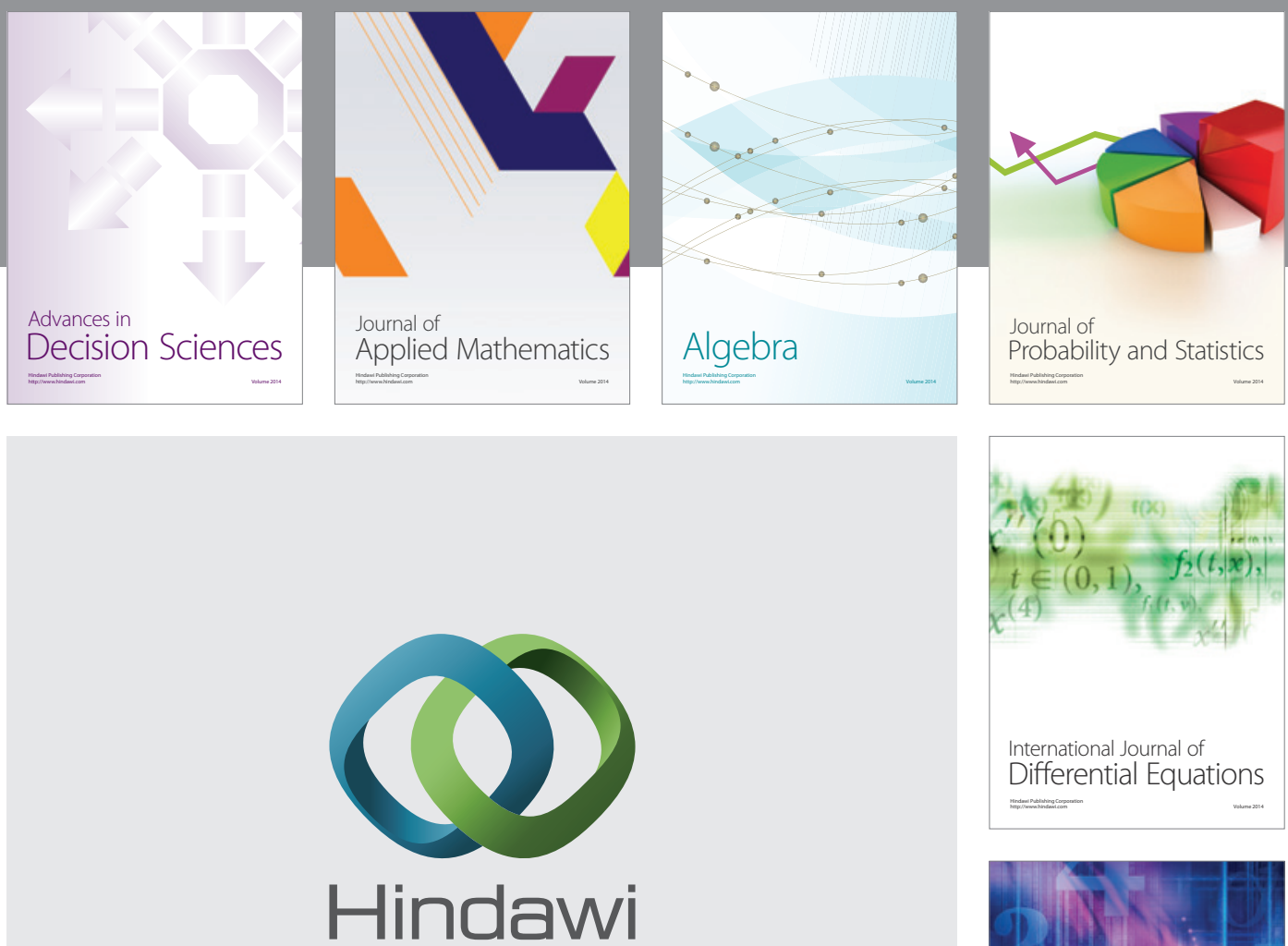

Submit your manuscripts at http://www.hindawi.com
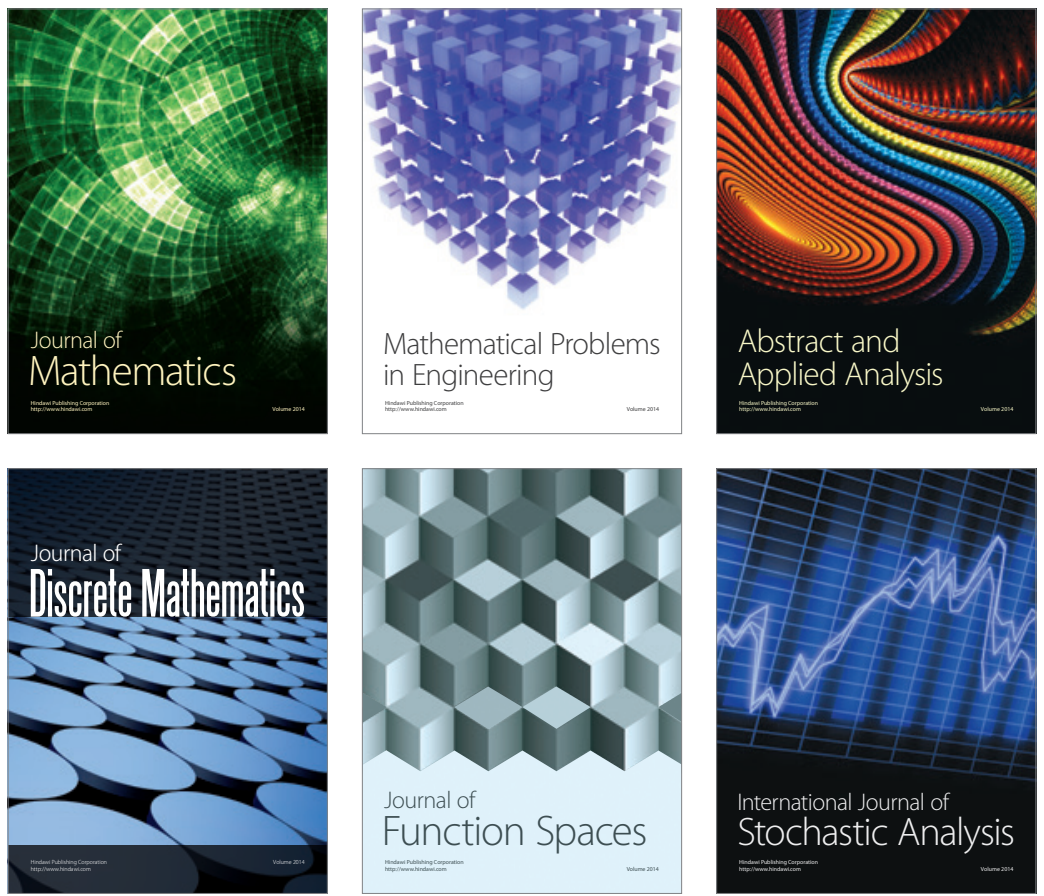

Journal of

Function Spaces

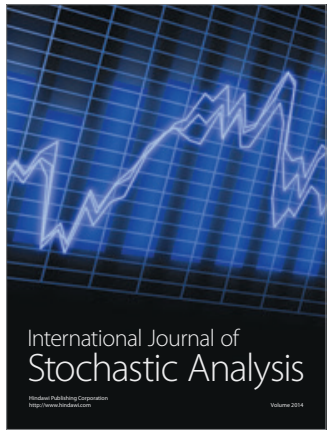

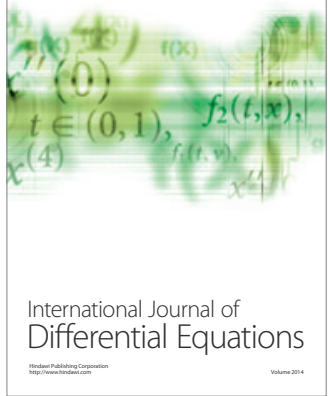
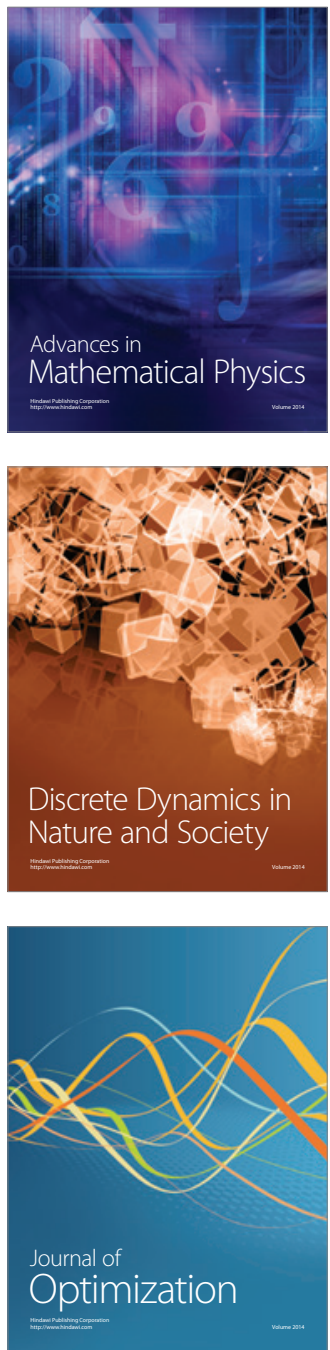